\title{
Positive Psychologie und ihre Bedeutung in der Beratung
}

\author{
Claudia Harzer • Heidi Möller
}

Online publiziert: 27. Juli 2017

(C) Springer Fachmedien Wiesbaden GmbH 2017

Heidi Möller: In der Beratungsliteratur wird zunehmend mehr auf die Konzepte der Positiven Psychologie gesetzt. Das war auch der Grund für uns, einen Schwerpunkt in der OSC zu setzen, der Einblicke in den aktuellen Stand dieses Konzepts ermöglicht. Wir wollen zeigen, was die Positive Psychologie überhaupt ist, wie sie sich historisch entwickelt hat und was die Berater/innen-Szene daraus lernen kann. In diesem Heft gehen wir der Frage nach, wie wirksam die Positive Psychologie ist und wann es Sinn macht, sich ihrer in Beratungszusammenhängen zu bedienen. Klar, dass ich als Psychoanalytikerin dies mit ambivalenten Gefühlen begleite; um ehrlich zu sein, setzen manche Schriften dieses Ansatzes sogar heftige Reaktanz in mir frei. Kannst Du als Positive Psychologin meine inneren Regungen verstehen? Warum kommen viele der Beiträge der Positiven Psychologie oft so missionarisch daher? Manchmal fühle ich mich dabei, als sei ich in der Kirche und höre einer Verkündigung zu.

Claudia Harzer: Ich bin schon häufiger mit ähnlichen Reaktionen konfrontiert worden und habe mir Gedanken darüber gemacht, warum es diese gibt. Ich vermute, dass es mehrere Ursachen gibt, die hier zusammenspielen.

Zum einen kann die Begriffswahl eine Ursache sein. Bedeutet das Label „Positive Psychologie“, dass ,der Rest“ der Psychologie „Negative Psychologie“ ist? So ist das Label „Positive Psychologie“ nicht gemeint. Es soll betonen, dass die Perspektive bewusst nicht defizitorientiert ist, sondern dass es um die empirische Erforschung

Dr. C. Harzer $(\bowtie)$

Institut für Psychologie, Technische Universität Darmstadt, Alexanderstraße 10, 64283 Darmstadt, Deutschland

E-Mail: kontakt@stark-im-job.org

Prof. Dr. H. Möller ( $\bowtie)$

Institut für Psychologie, Universität Kassel, Holländische Straße 36-38, 34127 Kassel, Deutschland

E-Mail: dr.heidi.moeller@t-online.de 
von internen (z. B. Persönlichkeitseigenschaften) und externen Bedingungen (z. B. soziale Unterstützung, schulische und berufliche Rahmenbedingungen) geht, die ein optimales Erleben und Funktionieren ausmachen, erhalten und fördern (z. B. Seligman und Csikszentmihalyi 2000).

Zum anderen ist Positive Psychologie als empirische Wissenschaft eine vergleichsweise junge Forschungsrichtung und muss die eigene Existenz und Notwendigkeit noch begründen und rechtfertigen. Offensive Rechtfertigungen können hier gegebenenfalls als unangenehm wahrgenommen werden, weil die bisherige Dominanz von defizitorientierten Ansätzen und Perspektiven stark betont und alternative Perspektiven vorgeschlagen werden.

Ferner rücken mit der voranschreitenden Forschungsaktivität der Positiven Psychologie neue Ansätze und Konstrukte in den Fokus, die die eigenen bisherigen Ansätze teils in Frage stellen. Das ist sicherlich eher unangenehm, weil dies zur Veränderung des eigenen Bildes von Psychologie und Beratung führen kann, und von Bekanntem verabschiedet man sich eher nicht gern. Wenn das Bewährte doch gut funktioniert, warum sollte man etwas verändern?

Generell sollte jedoch nicht vergessen werden, dass die Positive Psychologie stets als eine Ergänzung und nicht als Konkurrenz zu bestehenden psychologischen Disziplinen ins Leben gerufen wurde. Diese Sicht teile ich zu $100 \%$ und erkenne ausschließlich einen Mehrwert für die psychologische Forschungslandschaft darin.

Heidi Möller: Freud sprach davon, durch die analytische Kur neurotisches Elend in allgemeines Leid zu wandeln. Ist das nicht ein recht realistisches Ziel? Happy people überall, das kann ja nur eine Illusion sein. Zur Frage der Zielsetzung: Ich bin schon recht zufrieden, wenn Menschen mit ihren Schwierigkeiten besser umgehen können. Glück, das kann doch nur punktuelles Erleben sein. Bin ich aus Deiner Sicht eine Kulturpessimistin? Bin ich als psychodynamische Beraterin einem aus Deiner Sicht zu pessimistischem Menschenbild verhaftet und verhindere dadurch Entwicklung meiner Klienten?

Claudia Harzer: Auf diese Fragen hin schießen mir sehr viele Gedanken gleichzeitig durch den Kopf. Ich versuche sie mal zu sortieren:

Hier einmal der Versuch, das sehr komplexe menschliche Erleben in einem einfachen Modell auszudrücken: Man stelle sich vor, dass das menschliche Erlebensspektrum auf einem Kontinuum von -10 (z. B. sehr negativ, unangenehm oder krank) über 0 (neutral) bis hin zu +10 (z. B. sehr positiv, angenehm, gesund) beschreibbar ist. Je nach Ausgangslage sind meiner Meinung nach verschiedene Ansätze erfolgsversprechender. Ist die Ausgangslage von Klienten z. B. bei +2 , geht es ihnen nicht schlecht, aber sie suchen z. B. eine Beratung auf, weil sie zufriedener im Beruf sein möchten. In diesem Fall sind Ansätze der Positiven Psychologie geeignet, um das Ziel einer höheren Arbeitszufriedenheit genauer zu beleuchten und dessen Erreichung anzugehen. Geht es Klienten hingegen sehr schlecht, weil sie sich z. B. im Bereich von -8 befinden, sind Ansätze aus dem klinischen Bereich geeignet, um die Ursachen des Problems und dessen Reduzierung anzugehen. Nichtsdestoweniger glaube ich, dass Beratung mit Schwerpunkt auf Ansätzen der Positiven Psychologie sehr vom Wissen aus dem klinischen Bereich profitieren kann, aber auch umgekehrt. Weder die reine defizitorientierte noch die reine ressourcenorientierte Perspektive reichen aus, um das mögliche Erlebensspektrum vollständig abzudecken, aber eine 
gut gewichtete Mischung (,das gesunde Mittelmaß“) beider Perspektiven (vgl. den Beitrag von Michelle McQuaid in diesem Heft) kann dies.

Positive Psychologie ist nicht „Happiology“ (Peterson 2006). Es geht nicht darum, wie man Menschen den ganzen Tag lachen und in einer rosa-roten Wolke durchs Leben gehen lässt. Zielsetzung der positiv-psychologischen Forschung ist also nicht, wie man „happy people“ produziert, sondern die Erforschung dessen, was ein von optimalem Erleben und Funktionieren gekennzeichnetes Leben ausmacht. In den Medien wird das leider zu oft auf das Erleben von positiven Emotionen reduziert. Doch es geht um ein sehr viel breiteres Themenfeld (vgl. meinen Beitrag in diesem Heft), das Themen enthält wie das Erleben von Sinnhaftigkeit des eigenen Lebens, ausgeglichene Zufriedenheit (engl. contentment) und situationsangemessenes Nutzen eigener Talente und Stärken. Das Erleben von Glück (ein Gefühl mit hohem Arousal) ist also nicht das Zentrum oder ultimative Ziel positiv-psychologischer Forschung, sondern ein möglicher Aspekt, der ein von optimalem Erleben und Funktionieren gekennzeichnetes Leben ausmacht.

Zwei Arten von Interventionen (z. B. Seligman et al. 2005) sind gute Beispiele dafür, dass andere Ziele verfolgt werden, als nur das Glücksempfinden zu steigern: Eine Gruppe der Interventionen sind die sogenannten stärkenbasierten Interventionen, in denen sich die Personen mit ihren individuell wichtigsten positiven Eigenschaften (d.h. Charakterstärken) auseinandersetzen und sich im Sinne eines authentischen Verhaltens überlegen, wie sie diese auf neue Art und Weise z. B. im beruflichen Alltag einsetzen können. Studien zeigen, dass stärkenbasierte Interventionen etwa zu einer Erhöhung der Wahrnehmung des Berufs als Berufung, der Lebenszufriedenheit und der Bereitschaft zur persönlichen Weiterentwicklung (personal growth initiative) führen können (Harzer und Ruch 2016; Littman-Ovadia et al. 2014). Was diese Intervention bei Einzelpersonen auslöst und wie diese verarbeitet werden, zeigt der Beitrag von Markus Ebner in diesem Heft. Wie diese Interventionen im Rahmen eines Coachings eingebunden werden können, zeigt der Beitrag von Michelle McQuaid in diesem Heft.

Eine weitere Interventions-Übung, die im Rahmen positiv-psychologischer Forschung entwickelt wurde, ist die „Drei gute Dinge“-Übung. Ziel der Übung ist, jeden Abend vor dem Zu-Bett-Gehen den Tag Revue passieren zu lassen und drei Momente zu notieren, die angenehm waren. Wichtig hierbei ist, dass es um die kleinen alltäglichen Dinge (z. B. ein gutes Gespräch mit einem engen Freund; das fremde Kind, das man beim Entdecken eines Gänseblümchen beobachtet; vertieft einen spannenden Fachartikel gelesen) geht und nicht um die großen Ereignisse (z. B. Lottogewinn). Diese Übung kann helfen, die kleinen Dinge des Alltags mehr zu genießen. Studien zeigen, dass diese Übung zu einer Reduktion depressiver Symptome führen kann (z. B. Seligman et al. 2005).

Heidi Möller: Kritiker sprechen von der destruktiven Kraft positiven Denkens. Ist es nicht notwendig, auch negative Affekte zu spüren, sich mit Schmerz, Unlust, Enttäuschung zu konfrontieren, um in Veränderungsprozesse zu kommen? Sicherlich meine ich nicht dysfunktionales Grübeln, das nachweislich wenig bringt. Aber ohne Unangenehmes zu fühlen und dies genauer kennenzulernen, auszuwerten und Konsequenzen daraus zu ziehen, ohne ein wenig Leidensdruck setzen wir uns doch nicht in Bewegung, oder? 
Claudia Harzer: Dem stimme ich völlig zu. Es geht nicht um die Abschaffung von negativen Emotionen, da sie im Laufe der Evolution der Arterhaltung dienten (und immer noch dienen) und wichtige Informationsgeber sind (z. B. Fredrickson 1998, 2001). Die Forschung zur Rolle von positiven Emotionen war sehr lange sehr begrenzt, sodass ein Bedarf an Theorien zur Erklärung der Rolle positiver Emotionen vorhanden war und ist. In meinem Beitrag gehe ich auf eine Theorie (Fredricksons Broaden-and-Build-Theory) ein, welche die relevante Rolle positiver Emotionen zu erklären versucht. Auch hier geht es ausschließlich um die Ergänzung, nicht um die Abschaffung vorhandenen Wissens.

Heidi Möller: Zum Thema Ambivalenz: Viele Ansätze, wie z. B. das MI (Motivational Interviewing), wollen die Ambivalenz im Erleben beseitigen. Ich sehe nun gerade die Ambiguitätstoleranz als Entwicklungsziel an. Ist es nicht ein Ziel, mit einem „Dennoch“ zu leben? Ich liebe meinen Mann, obwohl er schnarcht; ich sehe meinen Vorgesetzten mit seinen Kompetenzen und Mängeln und lasse mich dennoch führen; ich hadere mit manchen Prozessen an der Universität und stehe dennoch jeden Morgen auf und arbeite an den Organisationszielen kräftig mit usw.

Claudia Harzer: Es trifft auf die Positive Psychologie als empirische Wissenschaft nicht zu, Ambivalenz im Erleben ,zu beseitigen“. Themen wie Ambiguitätstoleranz und Umgang mit Ambivalenz sind zumindest implizit enthalten. Beispiele hierfür sind in den untersuchten Persönlichkeitsmerkmalen zu finden. Persönlichkeitsmerkmale wie Tapferkeit und Urteilsvermögen behandeln den Umgang mit Ambivalenz und Ambiguität. Tapferkeit zeigt sich durch das Eintreten für eine Minderheitsmeinung, weil man sie für wichtig und richtig hält, selbst wenn man dafür mit sozialen Sanktionen rechnen muss. Urteilsvermögen bezieht sich darauf, Dinge zu durchdenken und von allen Seiten zu betrachten, sodass keine voreiligen Schlüsse gezogen werden und die eigene Meinung geändert werden kann, wenn es gute Gründe gibt.

Ich finde es bemerkenswert, dass Positive Psychologie mitunter Reaktanz auslöst, anstatt dass deren gewinnbringender Nutzen erkannt wird. Im Grunde genommen geht es in der Positiven Psychologie darum, dass psychische Gesundheit durch mehr als nur die Abwesenheit von Indikatoren psychischer Erkrankung beschrieben werden kann (World Health Organization 2014); und es geht darum, dass Forschung benötigt wird, um Indikatoren psychischer Gesundheit zu identifizieren und empirisch gut zu untermauern, sowie Interventionen zur Förderung dieser zu entwickeln. Wann welche Indikatoren sowie Interventions- und Therapieansätze besonders relevant sind, hängt unter anderem von der oben besprochenen Ausgangslage und den Zielen der Ratsuchenden ab.

Heidi Möller: Nun, liebe Leserinnen und Leser, machen Sie sich bitte selbst ein Bild. Claudia Harzer macht es Ihnen mit einem Überblick über die historische Entwicklung, dem Stand der Forschung und den wesentlichen Pfeilern des Konzepts der Positiven Psychologie leicht. Markus Ebner präsentiert eine Methodik, die 4-Evening-Questions zur unmittelbaren Anwendung in Ihrer Beratungspraxis. Und Michelle McQuaid zeigt in ihrem englischen Beitrag, dass durch die Art des Fragens im Coaching die Aufmerksamkeit der Klient/innen auf ihre persönlichen Stärken gelenkt und diese damit aktiviert werden können. Damit gehen wir neue Wege: In der 
OSC werden zukünftig auch von Zeit zu Zeit englischsprachige Originalbeiträge erscheinen, die uns lesenswert erscheinen.

Im offenen Teil des Heftes fasst Katrin Oellerich die Ergebnisse ihrer empirischen Untersuchung zur Frage der Coachingkultur vor. Der Coachingkultur-Forschungsansatz ist vergleichsweise neu, aber unverzichtbar. Wirksamkeitsforschung im Coaching fokussiert üblicherweise die Dyade Coach und Coachingpartner/in. Ohne eine günstige Coachingkultur aber, die Transferlernen ermöglicht, können selbst die Weltmeister der Branche nichts ausrichten.

In dem ersten Praxisbericht beschreibt Marga Löwer-Hirsch anschaulich, wie schwierig sich die Frage von Macht und Ohnmacht in Supervisionsprozessen oftmals stellt. Wie balancieren sich die Machtverhältnisse im Dreieck von Auftraggeber, Führungskräften und Teams. Ihr detailreicher Bericht fordert uns alle auf, eigene Erfahrungen von Macht- und Ohnmachtserfahrungen als Berater/innen zu reflektieren, und schärft den Blick für die Mikropolitik in Organisationen. Der zweite Praxisbericht bringt uns ein für viele eher ferneres Feld der Coachingpraxis näher: die Versicherungsbranche. Michael Schmitz skizziert die Herausforderungen, Paradoxien und Möglichkeiten der Beratung in diesem Bereich, den wir alle zumindest aus der Kund/innensicht kennen.

In einem Diskurs-Beitrag wendet sich Silja Kotte der Supervision von Coachingprozessen zu. Supervision wird von allen einschlägigen Berufsverbänden als das Mittel der Qualitätssicherung gepriesen. Was wissen wir aber über den Stand der Forschung, löst die Supervision von Coaches ihr Versprechen ein? Zudem wird ein praktikables Modell der Supervision vorgestellt. - An dieser Stelle dürfen Silja Kotte und ich Sie, lieber Leserinnen und Leser, bitten, weiter zur Fundierung dieser Qualitätssicherungsmaßnahme beizutragen. Beteiligen Sie sich doch rege an unserer Online-Befragung unter dem folgenden Link: https://www.soscisurvey.de/ QualitaetssicherungCoaching/.

Unsere Filmanalyse über den Film „Der Campus“ beschäftigt sich mit einer Organisation, die alle Leser/innen zumindest als „Kund/innen“ kennen, die Universität. Lothar Zechlin, langjähriger Rektor verschiedener Universitäten, beschreibt das Spannungsfeld zwischen drei Subkulturen, die in dieser Organisation zusammentreffen: die Gruppe der interessierten Gelehrten, die Kultur der politisierten Gruppenuniversität und die in der Verwaltung verkörperte Kultur des neutralen Rechtsstaats. „Wissenschaft, Politik und Recht könnten sich zwar gegenseitig inspirieren“, so schreibt er. Wie aber lassen sie sich unter Aufrechterhaltung ihre Eigenarten miteinander verbinden?

\section{Literatur}

Fredrickson, B.L. (1998). What good are positive emotions? Review of General Psychology, 2, 300-319. doi:10.1037/1089-2680.2.3.300.

Fredrickson, B.L. (2001). The role of positive emotions in positive psychology. American Psychologist, 56, 218-226. doi:10.1037//0003-066x.56.3.218.

Harzer, C., \& Ruch, W. (2016). Your strengths are calling: preliminary results of a strengths-based onlineintervention to increase calling. Journal of Happiness Studies, 17, 2237-2256. doi:10.1007/s10902015-9692-y. 
Littman-Ovadia, H., Lazar-Butbul, V., \& Benjamin, B.A. (2014). Strengths-based career counseling: overview and initial evaluation. Journal of Career Assessment, 22, 403-419. doi:10.1177/ 1069072713498483.

Peterson, C. (2006). A primer in positive psychology. Oxford: Oxford University Press.

Seligman, M.E.P., \& Csikszentmihalyi, M. (2000). Positive psychology: an introduction. American Psychologist, 55, 5-14. doi:10.1037//0003-066x.55.1.5.

Seligman, M., Steen, T., Park, N., \& Peterson, C. (2005). Positive psychology progress: empirical validation of interventions. American Psychologist, 60, 410-421.

World Health Organization (2014). Mental health: a state of well being. http://www.who.int/features/ factfiles/mental_health/en. Zugegriffen: 16. Juni 2017. 